\title{
Expression and clinical significance of miR-122 and miR-29 in hepatitis $B$ virus-related liver disease
}

\author{
T.J. Xing, D.F. Jiang, J.X. Huang and Z.L. Xu \\ Department of Infectious Diseases, Taizhou People's Hospital, Taizhou, China \\ Corresponding author: T.J. Xing \\ E-mail: xingtj518@sina.com
}

Genet. Mol. Res. 13 (3): $7912-7918$ (2014)

Received July 1, 2013

Accepted August 14, 2014

Published September 29, 2014

DOI http://dx.doi.org/10.4238/2014.September.29.4

\begin{abstract}
MicroRNA molecules have been increasingly regarded as a diagnostic and prognostic marker of certain diseases. The aim of this study was to investigate the expression and clinical significance of miR-122 and miR-29 in liver disease related to hepatitis B virus infection. The serum levels of miR-122 and miR-29 in 20 patients with hepatocellular carcinoma (HCC), 20 patients with liver cirrhosis (LC), 29 patients with chronic hepatitis B (CHB), 20 cases of hepatitis B virus carriers (ASC), and 20 healthy controls (HC) were determined by a fluorescence real-time quantitative PCR method and then evaluated by clinical correlation analysis. Compared with the serum levels of miR-122 in the HC, LC, and ASC groups, those in patients with HCC and CHB were significantly increased. The serum levels of miR-29 in LC patients were lower than those in the healthy controls $(\mathrm{P}<0.01)$. A positive correlation was observed between the expression of miR122 and miR-29, and HBV DNA in patients with CHB. A negative correlation was found between miR-29 and $\alpha$-fetoprotein in patients with HCC. The elevation in miR-122 was correlated with liver damage in CHB patients and with the pathogenesis of liver cancer in HCC patients. The decrease in miR-29 expression was related to the incidence
\end{abstract}


of liver fibrosis. The detection of miR-122 and miR-29 may be useful in evaluating the inflammatory liver injury and fibrosis associated with chronic HBV infection.

Key words: Chronic hepatitis B; Hepatocellular carcinoma; miR-122; miR-29

\section{INTRODUCTION}

Hepatitis B virus (HBV) infection is a serious public health problem. There are currently 350 million cases of $\mathrm{HBV}$ infection in the world, with approximately 100 million HBV cases and more than 20 million hepatitis B patients in China (Ganem and Prince, 2004; Lu and Zhuang, 2009). Approximately 20 to $40 \%$ of these cases develop into cirrhosis or liver cancer because of repeated episodes of chronic inflammation. Treatment and prognosis are poor once liver cirrhosis or cancer has formed, making early diagnosis and treatment crucial to improving efficacy and prognosis. Regular hepatocellular carcinoma (HCC) surveillance by ultrasound scanning and/or $\alpha$-fetoprotein (AFP) levels may identify early cancers and reduce mortality (Wong et al., 2008). However, more than $30 \%$ of patients do not receive early diagnosis. The search for markers of early diagnosis remains an urgent problem for further exploration.

MicroRNAs (miRNAs) are approximately 22-nucleotide non-coding RNAs that regulate target genes at post-transcriptional levels. They not only play an important role in cell development, differentiation, and physiological function, but are also significant in the development of tumors, viral infections, and other closely related diseases (Bartel, 2004; Dykxhoorn and Lieberman, 2005). Furthermore, miRNA molecules have been increasingly regarded as a diagnostic and prognostic marker in the evaluation of certain diseases. Recent studies have found that miRNAs are abundant in the liver and modulate a diverse range of liver functions. Several miRNAs in serum and tissue have been reported in the diagnosis and prognosis of tumors related to HBV infection (Li et al., 2010; Qi et al., 2011).

Circulating miRNAs are emerging as promising biomarkers for several pathological conditions. Some reports have shown that the profiles of serum miRNA expression can specifically predict liver injury caused by chronic hepatitis B (CHB) (Chen et al., 2008; Zhou et al., 2011). MiRNA-122 family is the most abundant type of miRNA in the liver (Lewis and Jopling, 2010). Changes in miRNA-122 are associated not only with the pathogenesis of $\mathrm{HCC}$, but also with liver damage. Hepatic fibrosis is influenced by several epigenetic factors that control wound-healing responses. MiRNA-29 family is one of the most important types of RNA involved in the healing process (Roderburg et al., 2011). Therefore, to explore the application value of miR-122 and miR-29 in the evaluation of CHB and related liver disease, this study determined the serum levels of miR-122 and miR-29 expression in different types of chronic HBV infection and related liver disease, as well as analyzing such expression with clinical indicators.

\section{MATERIAL AND METHODS}

\section{Subjects}

Blood samples were collected from 69 cases of chronic HBV infection and 20 healthy 
controls (HC) at Taizhou People's Hospital from June 2011 to October 2012. Informed consent was obtained from all the subjects. Among the 69 cases, 29 had CHB; the gender ratio was 24 males to 5 females, and the mean age was $37 \pm 11$ years. Among the total number of cases, 20 were chronic HBV carriers (ASC), among which 9 were males and 11 females, with an average age of $39 \pm 11$ years. Furthermore, 20 had liver cirrhosis (LC), among which 15 were males and 5 females, with mean age of $52 \pm 13$ years. The diagnostic criteria were based on the 2010 Chronic Hepatitis B Prevention Guide of China (Chinese Liver Disease Association, 2011). All patients tested negative for antibodies against hepatitis A, C, D, and E viruses, as well as against HIV. All patients with histories and clinical features of drug-induced liver injury, alcoholic hepatitis, and steatohepatitis, as well as those treated with nucleotide/nucleotide analog antiviral or immunomodulatory drugs in the preceding 6 months, were excluded. Among the 20 patients with HCC, 16 were males and 4 females, with mean age of $57 \pm 10$ years. Among the $20 \mathrm{HC}$ cases in our hospital, 11 were males and 9 females, with a mean age of $34 \pm 12$ years. The experimental protocol was approved by the Ethics Committee of Taizhou People's Hospital.

\section{Determination of serum miR-122 and miR-29 by real-time quantitative PCR}

The levels of miR-122 and miR-29 expression were determined by the SYBR Green Dye assay. Serum total RNA was extracted with Trizol (Invitrogen Company, USA) in a one-step extraction procedure. Hsa-miR-122, miR-29, and U6 reverse transcription primers and amplification primers were designed and synthesized by Shanghai Sangon Biological Engineering Co. Ltd. The specific sequences are presented in Table 1. cDNA synthesis was conducted with reverse transcription. The reaction system of $20 \mu \mathrm{L}$ was as follows: $2 \mu \mathrm{L}$ dNTP, $2 \mu \mathrm{L}$ 10X RT Buffer, $0.3 \mu \mathrm{L}$ RT-specific primers, $200 \mathrm{ng}$ total RNA, $0.1 \mu \mathrm{L}$ Moloney Murine Leukemia Virus reverse transcriptase, $0.2 \mu \mathrm{L}$ RNase inhibitor, and no RNA enzyme water. The reaction conditions were $16^{\circ} \mathrm{C}$ for $30 \mathrm{~min}, 42^{\circ} \mathrm{C}$ for $40 \mathrm{~min}$ and $85^{\circ} \mathrm{C}$ for $5 \mathrm{~min}$. PCR was completed with a real-time fluorescent quantitative PCR instrument (ABI 7300; Applied Biosystems, Foster City, CA, USA) under the following conditions: $95^{\circ} \mathrm{C}$ for $2 \mathrm{~min}$ and 40 cycles of $95^{\circ} \mathrm{C}$ for $10 \mathrm{~s}$ and $60^{\circ} \mathrm{C}$ for $40 \mathrm{~s}$. U6 was used as reference. Data were analyzed using the $2-\Delta \Delta \mathrm{Ct}$ method. $\Delta \mathrm{Ct}=$ miR122 (29) $\mathrm{Ct}$ - U6 $\mathrm{Ct}$ (the higher the $\Delta \mathrm{Ct}$ value, the lower the expression).

\begin{tabular}{|c|c|c|}
\hline Gene name & Primer sequence & Product (bp) \\
\hline U6 & $\begin{array}{l}\text { F: 5'-GCTTCGGCAGCACATATACTAAAAT-3' } \\
\text { R: 5'-CGCTTCACGAATTTGCGTGTCAT-3' }\end{array}$ & 89 \\
\hline Hsa-miR-122 & $\begin{array}{l}\text { GSP: 5'-ACACTCCAGCTGGGTGGAGTGTGACAATCC-3' } \\
\text { R: 5'-TGGTGTCGTGGAGTCG-3' }\end{array}$ & 66 \\
\hline Hsa-miR-29 & $\begin{array}{l}\text { GSP: 5'-ACACTCCAGCTGGGTAGCACCATCTGAAAT-3' } \\
\text { R: 5'-TGGTGTCGTGGAGTCG-3' }\end{array}$ & 66 \\
\hline
\end{tabular}

\section{Determination of HBV DNA and serum markers}

The levels of HBV DNA were determined by fluorescence quantitative PCR (lower detection limit of $10^{3}$ copies $/ \mathrm{mL}$; Applied Biosystems). HBV PCR fluorescence quantitative detection kits were purchased from the Shanghai branch of Biological Engineering Company 
Ltd. The serum markers of HBV, anti-HAV, anti-HCV, anti-HDV, and anti-HEV were detected by ELISA. Biochemical markers were assayed using a biochemical automatic analyzer. AFP was detected by chemiluminescence determination.

\section{Statistical analysis}

All values are reported as means \pm SD. One-way ANOVA, followed by the StudentNewman-Keuls- $q$ test, was used to evaluate differences between the groups. Alanine aminotransferase (ALT), AFP, and HBV DNA levels exhibited a skewed distribution and were logarithmically transformed. The two groups were compared by using the two-sample $t$-test. The correlation analysis was conducted using Pearson's correlation. Data analysis was performed using the SPSS17.0 statistical software (SPSS, Inc.). P $<0.05$ was considered to be statistically significant.

\section{RESULTS}

\section{Determination of miR-122 expression in serum of different populations}

With the RT-PCR method, serum levels of miR-122 were determined in different types of chronic HBV infection related to liver disease. The results showed that the levels of miR-122 expression were significantly increased in patients with HCC and CHB compared with those in the $\mathrm{HC}$ cases. The difference was statistically significant $(\mathrm{P}=0.000)$. The expression levels of miR-122 in serum of patients with HCC and CHB were significantly higher than those in the LC and ASC groups. The difference was also statistically significant $(\mathrm{P}=$ $0.000)$. No statistically significant difference was found between the LC and ASC groups (Table 2).

Table 2. Comparison of miR-122 and miR-29 expression in serum of different populations.
\begin{tabular}{lccc}
\hline Group & $\mathrm{N}$ & miR-122 $(\Delta \mathrm{Ct})^{*}$ & miR-29 $(\Delta \mathrm{Ct})^{*}$ \\
\hline HCC & 20 & $8.29 \pm 2.41$ & $7.34 \pm 1.38$ \\
LC & 20 & $9.73 \pm 1.89$ & $8.07 \pm 0.97$ \\
CHB & 29 & $7.57 \pm 2.34$ & $5.64 \pm 1.21$ \\
ASC & 20 & $9.94 \pm 2.36$ & $7.46 \pm 0.71$ \\
HC & 20 & $11.24 \pm 2.26$ & $7.06 \pm 1.30$ \\
\hline
\end{tabular}

${ }^{*} \mathrm{~F}=9.36 ; \mathrm{P}=0.000 ;{ }^{*} \mathrm{~F}=14.94 ; \mathrm{P}=0.000 . \mathrm{HCC}=$ hepatocellular carcinoma $\mathrm{LC}=$ liver cirrhosis $\mathrm{CHB}=$ chronic hepatitis $\mathrm{B}$; $\mathrm{ASC}=$ chronic $\mathrm{HBV}$ carriers; $\mathrm{HC}=$ healthy controls.

\section{Determination of miR-29 expression in serum of different populations}

Similarly, the RT-PCR method was used to detect the serum levels of miR-29 in different types of chronic HBV infection related to liver disease. The levels of serum miR-29 expression were significantly decreased compared with those in the HC and LC groups; in the CHB group the level was significantly increased, with a statistically significant difference (P $=0.000$ ). The levels of serum miR-29 expression in the HCC, LC, and ASC groups were significantly lower than in the CHB group, with a statistically significant difference $(\mathrm{P}=0.000)$ (Table 2). 


\section{Correlation of serum miR-122, miR-29, and clinical indicators of patients with CHB}

The ALT level of 29 patients with CHB was $2.21 \pm 0.48$ (logarithm values) and the HBV DNA level was $6.28 \pm 1.64$ (logarithm values). No significant correlation was found between the levels of miR-122, miR-29, and ALT. The levels of miR-122 and miR-29 were positively correlated with HBV DNA $(r=0.64,0.44, \mathrm{P}=0.000,0.017)$, as shown in Figure $1 \mathrm{~A}$ and $\mathrm{B}$.

A

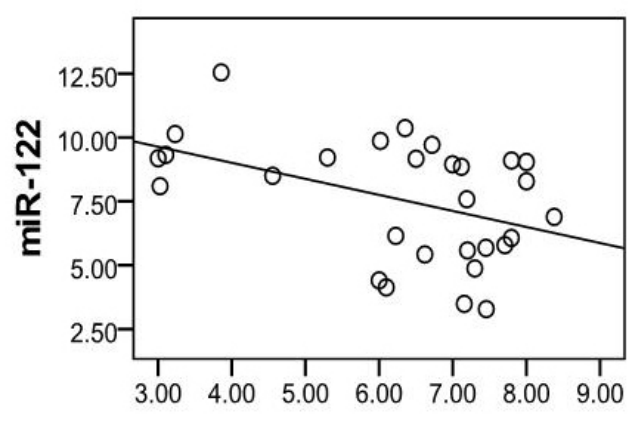

HBV DNA

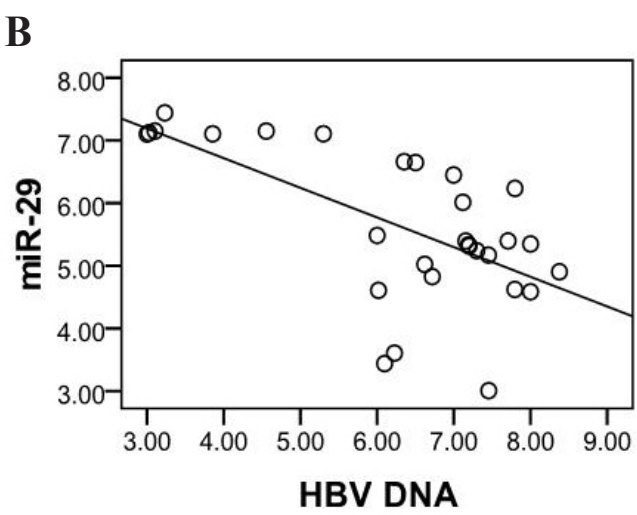

Figure 1. Correlation between miR-122, miR-29 and HBV DNA of patients with chronic hepatitis B. A. miR-122; B. $\operatorname{miR}-29$.

\section{Correlation of serum miR-122, miR-29, and clinical indicators of patients with HCC}

The 20 HCC patients had an ALT level of $1.50 \pm 0.36$ (logarithm values) and an AFP level of $2.17 \pm 0.81$ (logarithm values). No significant correlation was found between miR-122, ALT, and AFP. No significant correlation was found between miR-29 and ALT. A negative correlation was found between the levels of miR-29 and AFP $(r=-0.48, \mathrm{P}=0.031)$, as shown in Figure 2.

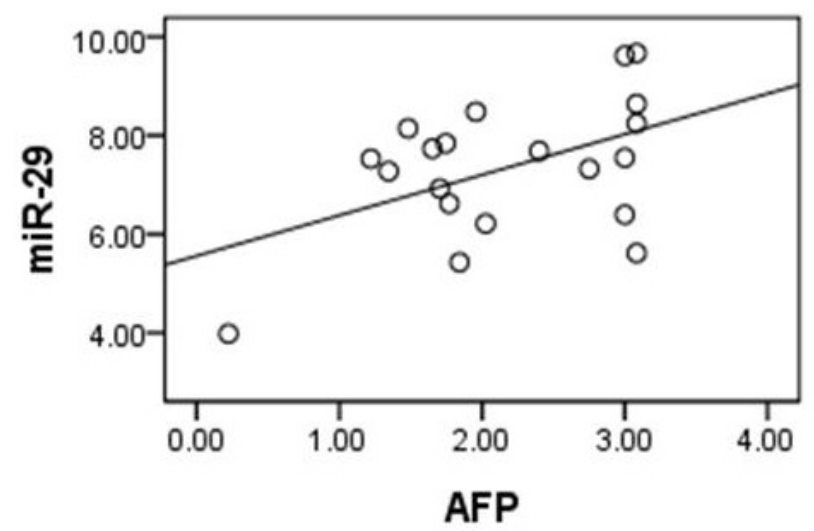

Figure 2. Correlation between miR-29 and $\alpha$-fetoprotein (AFP) of patients with hepatocellular carcinoma. 


\section{Comparison of ALT levels of CHB and HCC patients}

The ALT levels of 29 patients with CHB and 20 patients with HCC were $2.21 \pm 0.48$ and $1.50 \pm 0.36$ (logarithm values), respectively. The former was significantly higher than the latter, and the difference was statistically significant $(t=5.68, \mathrm{P}=0.000)$.

\section{DISCUSSION}

Chronic HBV infection can alter miRNA molecule expression, particularly the liverspecific expression patterns of miRNA. These changes may be associated with persistent chronic infection and disease progression. Clarifying these variations not only helps elucidate the mechanism of chronic HBV infection, but also provides new molecular markers for clinical diagnosis and the evaluation of antiviral treatment efficacy. Zhang et al. (2010) found that serum miR-122 expression can be used as a marker of liver tissue damage in patients with chronic HBV infection, with good sensitivity and specificity. Xu et al. (2011) reported that serum miR-21, miR-122, and miR-223 were increased in patients with chronic HBV infection and liver cancer, and that these can be used as specific markers of liver damage, but not specific to liver cancer. The results of the present study showed that the serum levels of miR-122 in patients with HCC and CHB were significantly higher than those in the serum of patients in the LC, ASC, and $\mathrm{HC}$ groups, suggesting that the expression of miR-122 is related to liver injury caused by CHB. No significant difference was found between the miR-122 expressions of the HCC and CHB groups. However, the ALT levels in patients with CHB were significantly higher than those in patients with HCC, suggesting that the mechanism of the elevation of miR-122 levels may not be the same in the serum of patients in the HCC and CHB groups. This finding warrants further investigation.

Liver fibrosis results from the body's inflammatory reaction and necrosis at the tissue injury site. The occurrence of liver fibrosis or cirrhosis often accompanies the process of CHB virus infection. Several studies have shown that the abnormal expression of miRNA molecules has a significant function in the development of liver fibrosis. Roderburg et al. (2011) found a panel of miRNAs that were specifically regulated in the liver of mice suffering from hepatic fibrosis. Among them, all three members of the miR-29 family were significantly downregulated in the liver of $\mathrm{CCl}_{4}$-treated mice. The specific regulation of miR-29 members in murine fibrosis models was correlated with the low expression of miR-29 in the liver of patients with advanced liver fibrosis. The results of the current study demonstrated that the levels of serum miR-29 expression in patients with LC significantly decreased compared with those in HC cases and CHB patients, suggesting that the downregulation of miR-29 is associated with liver fibrosis. Hepatic stellate cell activation is the central link of liver fibrosis. Several studies have shown that the expression of miR-150 and miR-194 is significantly downregulated, and let-7 family members are significantly increased in the hepatic stellate cells of the BDL mouse model of liver fibrosis. The overexpression of miR-150 and miR-194 can inhibit the proliferation of hepatic stellate cells and type I collagen reduction (Venugopal et al., 2010). A previous study found that the overexpression of miR-29 in the stellate cells in mice reduced collagen fiber levels, suggesting that miR-29 also affects the function of hepatic stellate cells and is involved in the regulation of liver fibrosis (Roderburg et al., 2011).

Waidmann et al. (2012) showed that the levels of miRNA-122 expression during chronic HBV infection were positively correlated with serum ALT, HBV DNA, and HBsAg expres- 
sion levels, and can distinguish between high and low risks of disease progression in patients. In the present research, no correlation was found between the expressions of miR-122 and ALT in the serum of patients with CHB. The differences may be attributed to the study population, detection methods, and other relevant factors. The levels of miR-122 and miR-29 were positively correlated with HBV DNA in patients with CHB. The replication of HBV was inhibited by miR-122. Whether miR-29 was affected by the replication of HBV DNA requires further exploration. The correlation analysis indicated that serum miR-29 was negatively correlated with AFP in patients with HCC, suggesting that miR-29 has a specific function in the pathogenesis of liver cancer. However, this finding requires confirmation with empirical evidence.

In summary, the results of this study indicated that an elevated expression of miR122 may be associated with the occurrence of liver damage in CHB and liver cancer, especially in the former. Low miR-29 expression may be related to liver fibrosis. The detection of miR-122 and miR-29 may be useful in evaluating the liver injury and fibrosis associated with HBV infection.

\section{Conflicts of interest}

The authors declare no conflict of interest.

\section{REFERENCES}

Bartel DP (2004). MicroRNAs: genomics, biogenesis, mechanism, and function. Cell 116: 281-297.

Chen X, Ba Y, Ma L, Cai X, et al. (2008). Characterization of microRNAs in serum: a novel class of biomarkers for diagnosis of cancer and other diseases. Cell Res. 18: 997-1006.

Chinese Liver Disease Association (2011). Chinese Liver Disease Association and Chinese Medical Association Infectious Disease Branch. Chronic Hepatitis B Prevention Guide (2010 edn.). Chin. J. Hepatol. 19: 13-24.

Dykxhoorn DM and Lieberman J (2005). The silent revolution: RNA interference as basic biology, research tool, and therapeutic. Annu. Rev. Med. 56: 401-423.

Ganem D and Prince AM (2004). Hepatitis B virus infection - natural history and clinical consequences. N. Engl. J. Med. 350: 1118-1129.

Lewis AP and Jopling CL (2010). Regulation and biological function of the liver-specific miR-122. Biochem. Soc. Trans. 38: 1553-1557.

Li LM, Hu ZB, Zhou ZX, Chen X, et al. (2010). Serum microRNA profiles serve as novel biomarkers for HBV infection and diagnosis of HBV-positive hepatocarcinoma. Cancer Res. 70: 9798-9807.

Lu FM and Zhuang H (2009). Management of hepatitis B in China. Chin. Med. J. 122: 3-4.

Qi P, Cheng SQ, Wang H, Li N, et al. (2011). Serum microRNAs as biomarkers for hepatocellular carcinoma in Chinese patients with chronic hepatitis B virus infection. PLoS One 6: e28486.

Roderburg C, Trautwein C and Luedde T (2011). MicroRNA-199a/b-3p: a new star in the liver microcosmos. Hepatology 54: 729-731.

Venugopal SK, Jiang J, Kim TH, Li Y, et al. (2010). Liver fibrosis causes downregulation of miRNA-150 and miRNA-194 in hepatic stellate cells, and their overexpression causes decreased stellate cell activation. Am. J. Physiol. Gastrointest. Liver Physiol. 298: G101-G106.

Waidmann O, Bihrer V, Pleli T, Farnik H, et al. (2012). Serum microRNA-122 levels in different groups of patients with chronic hepatitis B virus infection. J. Viral Hepat. 19: e58-e65.

Wong GL, Wong VW, Tan GM, Ip KI, et al. (2008). Surveillance programme for hepatocellular carcinoma improves the survival of patients with chronic viral hepatitis. Liver Int. 28: 79-87.

Xu J, Wu C, Che X, Wang L, et al. (2011). Circulating microRNAs, miR-21, miR-122, and miR-223, in patients with hepatocellular carcinoma or chronic hepatitis. Mol. Carcinog. 50: 136-142.

Zhang Y, Jia Y, Zheng R, Guo Y, et al. (2010). Plasma microRNA-122 as a biomarker for viral-, alcohol-, and chemicalrelated hepatic diseases. Clin. Chem. 56: 1830-1838.

Zhou J, Yu L, Gao X, Hu J, et al. (2011). Plasma microRNA panel to diagnose hepatitis B virus-related hepatocellular carcinoma. J. Clin. Oncol. 29: 4781-4788. 